\section{The Use of a Stereovision System in Shape Detection of the Side Surface of the Body of the Mining Machine Working Unit}

\author{
Piotr Cheluszka, Amadeus Jagieła-Zając \\ Silesian University of Technology, Poland
}

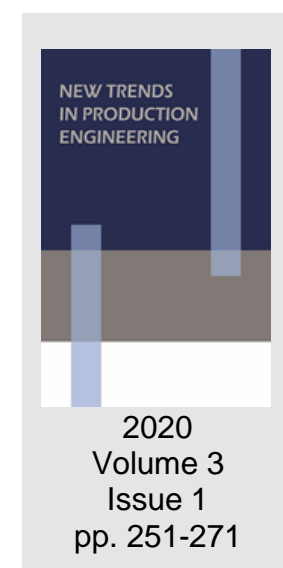

Date of submission to the Editor: 02/2020

Date of acceptance by the Editor: 03/2020

\title{
INTRODUCTION
}

Knowledge of the actual shape of the side surface of the working units of mining cutting machines underlies the robotization of the pick holders assembly process following the design assumptions (Dolipski, 2010; Cheluszka \& Nocoń, 2015). If the body of the cutting head/cutting drum is recovered during the overhaul process, its dimensions usually do not match the nominal dimensions. In the case of robotic production stations, real-time measurement of the distance between the pick holder base and the side surface of the cutting head/cutting drum of the mining machine while passing it to the side surface (Fig. 1) gives the possibility of automatic adjustment of the pick holder in order to:

- allow welding of the pick holder to the side surface,

- prevent the pick holder from colliding with the side surface of the cutting head/cutting drum.

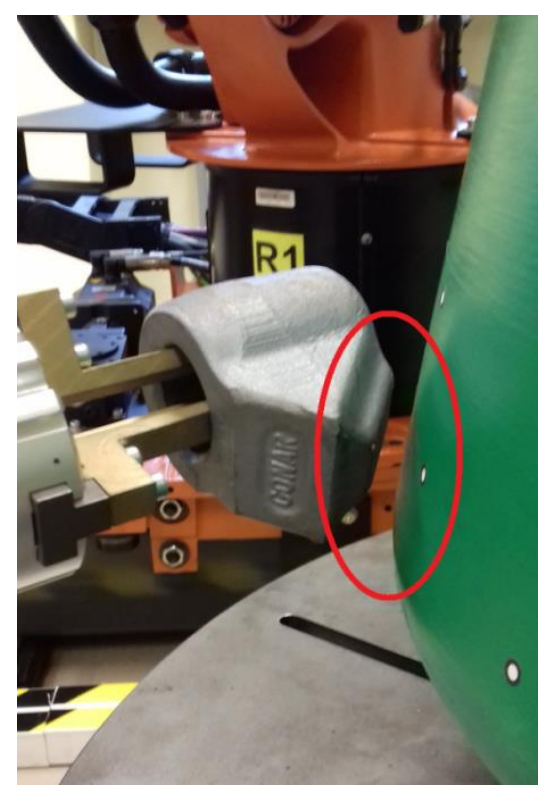

Fig. 1 Measured distance

Source: (Jagieła-Zając and Cheluszka, 2019) 
In the measuring method proposed in the previous work, in order to determine the distance between the pick holder base and the side surface of the cutting head, the KUKA VisionTech stereovision vision system, consisting of two cameras in a convergent orientation was used (Fig. 2).

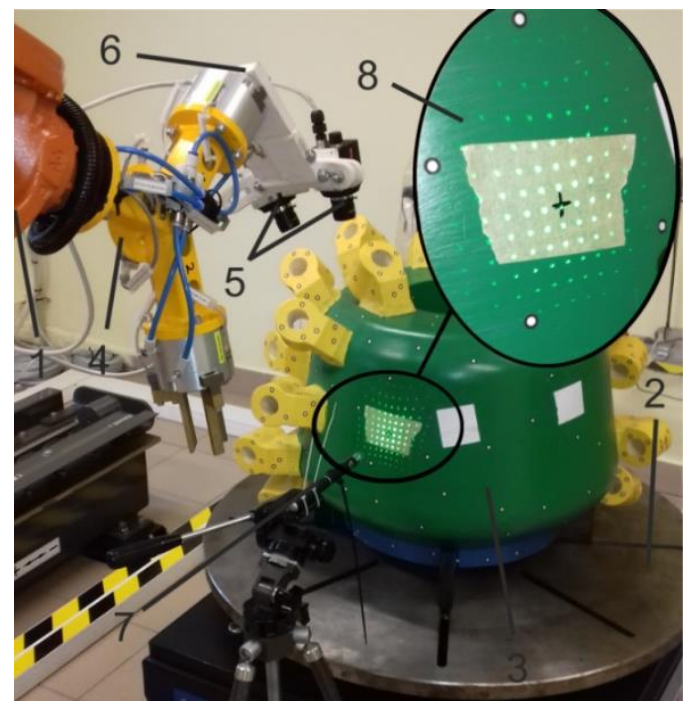

Fig. 2 Experimental stand in the robotics laboratory of the Department of Mining Mechanization and Robotization:

1 - robot KUKA KR 16-2, 2- positioner PEV-1-2500, 3 - side surface of the cutting head, 4 - gripper, 5 - KUKA MXG20 cameras, 6 - special construction bracket, 7 - projection device, 8 - a marker grid displayed on the side surface of the cutting head

The vision system takes photos while the robot is positioning the pick holder. The advance direction of the gripper coincides with the direction perpendicular to the pick holder base. Cameras should be located in a specific place both in relation to the base of the pick holder and the side surface of the cutting head/cutting drum so that it is possible to record all points of interest. These points are the corners of the pick holder base and the marker mesh applied to the side of the cutting head/cutting drum by the projection device (Goral, 2014). The location of the pick holder base in space is determined by the position of the TCP point of the robot tool positioning the pick holder and the orientation of the coordinate system axis associated with this tool. This work focuses on the issue of identifying (extracting) markers applied to the side surface of the cutting head/cutting drum on recorded images (Yang, Zhang \& Zhang, 2019). The applied grid should be as dense as possible and markers as small as possible so that the quantization results of the data contained in the images have a sufficient number of levels of representation, which increases the measurement accuracy (Zorski \& Samsel, 2009). The problem of recognition and indexation of markers in the measurement photos will be presented on the example of the cutting head of a boom-type roadheader.

\section{AUTOMATIC MARKER IDENTIFICATION METHOD}

The use of automatic detection of markers applied to the side surface of the cutting head of a roadheader determines the need to identify individual points on a pair of acquired photos (Ewald, 2009). During the reconstruction of the 
three-dimensional scene during the triangulation process, it is necessary to know the location of each of the markers on both measurement photos (Corke, 1996; Kryjak \& Komorkiewicz, 2013). Identification of individual markers of pattern applied on photos reduces the amount of processed information to a minimum by extracting only relevant information for further processing of photos (Raviya, Kothari and Vyas, 2014). The process of automatic marker identification is described by the algorithm shown in Fig. 3.

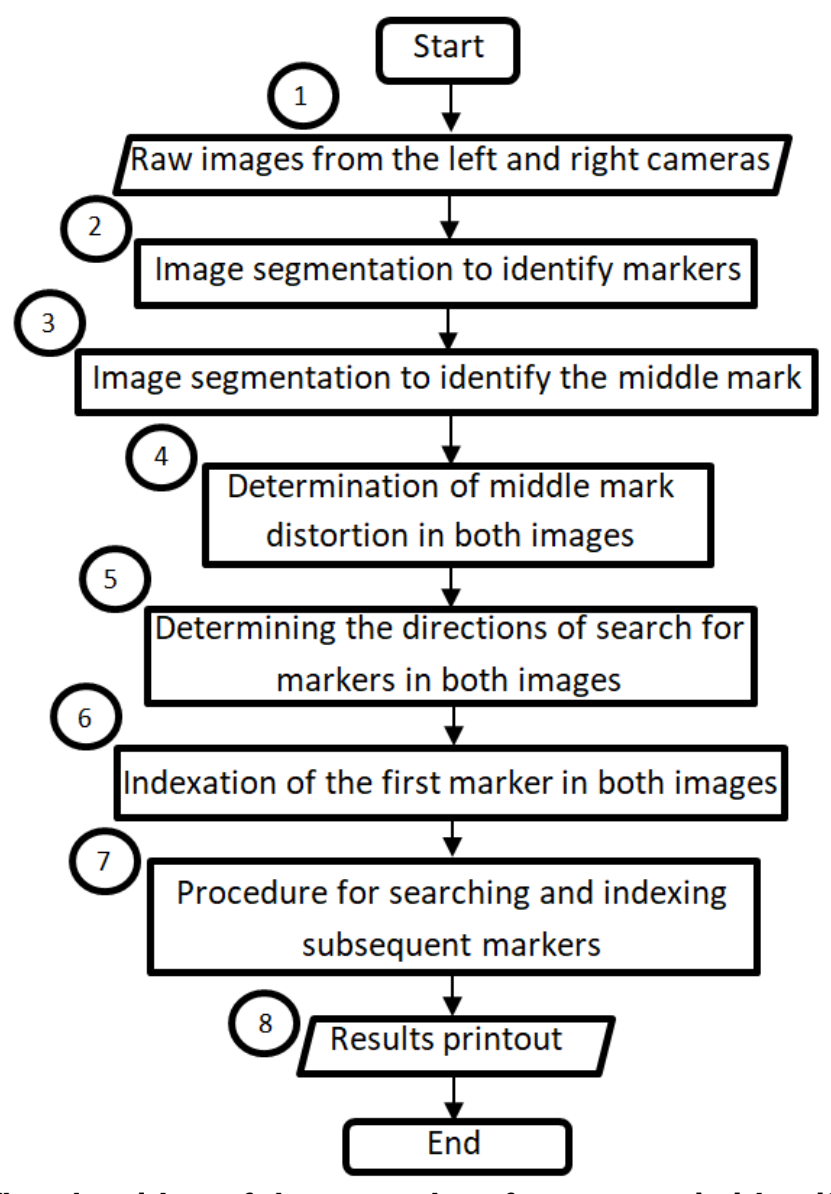

Fig. 3 The algorithm of the procedure for automatic identification of markers on measuring photos

It can be divided into three stages:

- image segmentation to extract the grid pattern applied to the side surface of the cutting head and the middle mark,

- determining the pattern distortion and determining the direction of marker search,

- recognizing and indexing pairs of markers in the pictures.

The Image Processing Toolbox of the Matlab environment was used for image processing and analysis (Wróbel \& Koprowski, 2004). As a result of the acquisition of measurement photos, two images of the scene are obtained, in which the body of the cutting head and markers applied to the side surface of the head are located (Fig. 4). The objects in the measurement photos, due to the convergent camera system used, have different orientation relative to the axis of the coordinate systems assigned to individual photos (Zhang, 2009). 


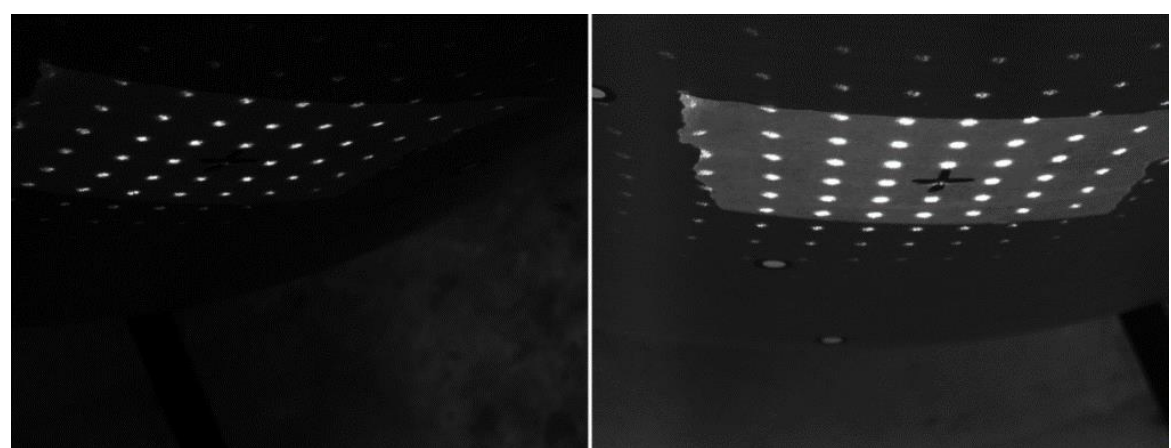

Fig. 4 Raw photos taken during measurements:

a) from the left camera, b) from the right camera

Before starting the spatial reconstruction, it is necessary to index individual pairs of markers contained in the pictures (Wojciechowski \& Bal, 2012).

The pictures recorded by the cameras are monochrome. After loading them into Matlab, they are pre-processed - they are converted into eight-bit images in shades of gray. In order for the analyzed pair of images to have a similar intensity (brightness) value, the imadjust function changes the image brightness levels (Fig. 5).
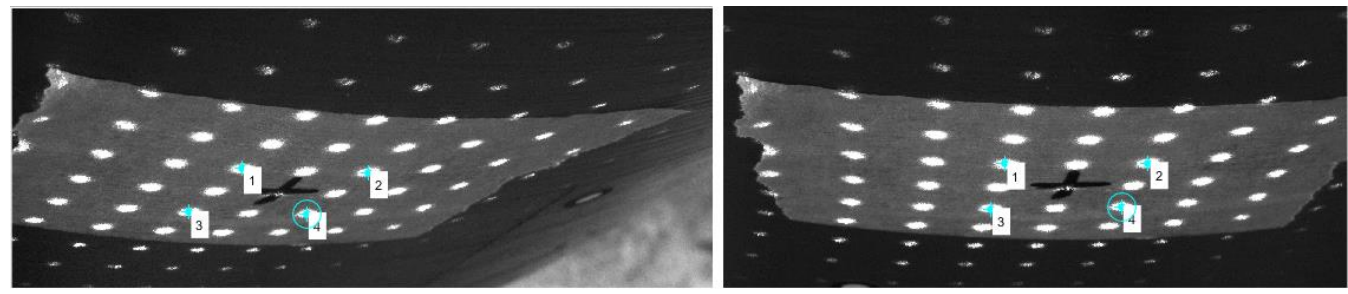

Fig. 5 Application of the imadjust function - the corresponding dots are visible in the pictures: a) from the left camera, b) from the right camera

The operation called histogram stretching adjusts the contrast of the images so that $1 \%$ of the data is saturated at low and high intensity. This is described by the equation (Kubiak, 2014):

where:

$$
m(g)=\frac{255}{\max -\min }(g-\min )
$$

max - maximum pixel brightness value present in the source image,

min - minimum pixel brightness value present in the source image,

$m(g)$ - resulting pixel brightness value $m$,

$g$ - input brightness value of a given pixel.

During the acquisition of photos with cameras, despite ensuring the same lighting conditions, the contrast values on the images downloaded with them differ. The analyzed photos are subject to distortions caused by optical system defects (distortion). The undistortimage function removes these distortions based on data from the calibration of the vision system (Bräuer-Burchardt \& Voss, 2000).

\section{IMAGE SEGMENTATION}

First, images of markers applied to the side surface of the cutting head are extracted on a pre-processed pair of photos. Scene background extraction 
is obtained as a result of successive morphological operations: erosion and dilation (Fig. 6) \& (Fig. 7).

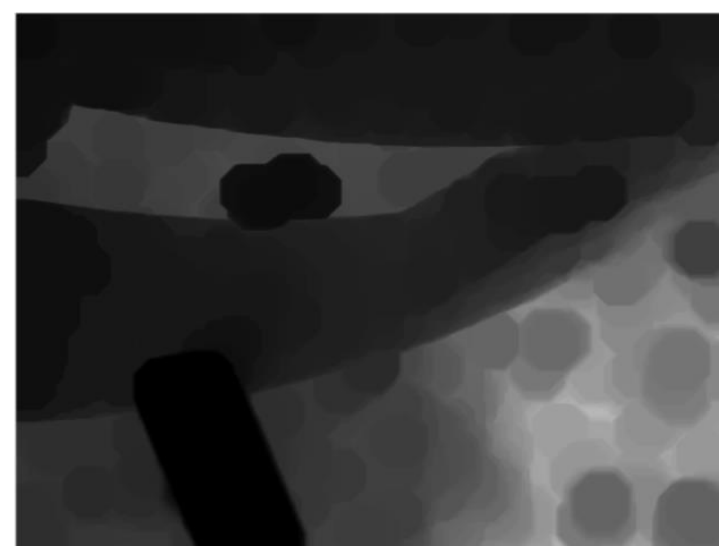

Fig. 6 The result of applying erosion on monochrome photographs

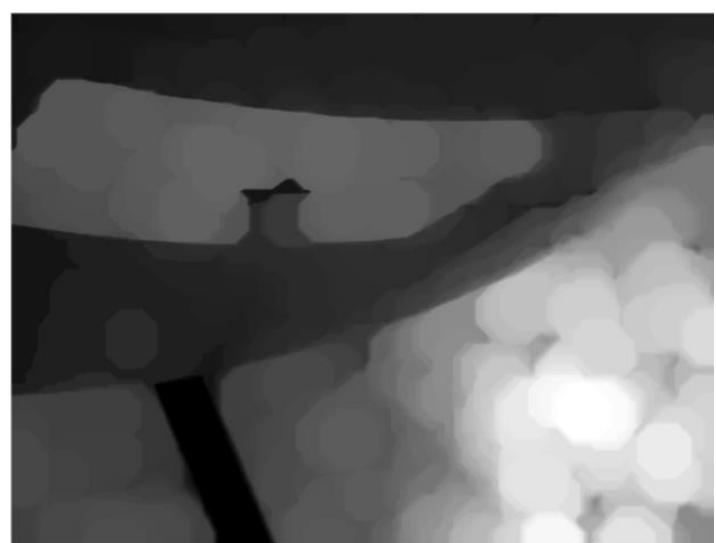

Fig. 7 The result of applying dilation on monochrome photographs

This is called opening the object (Sricha \& Khan, 2013). The size and shape of the kernel (mask, structuring element) used in morphological operations are determined by the size of the objects (markers) in the image, which depends on the type of projection device. As a result of subtracting the separated background from the pre-processed image, markers become more prominent in the newly created image (Fig. 8). This operation is called a Top-Hat operation (Fisker et al., 1998).

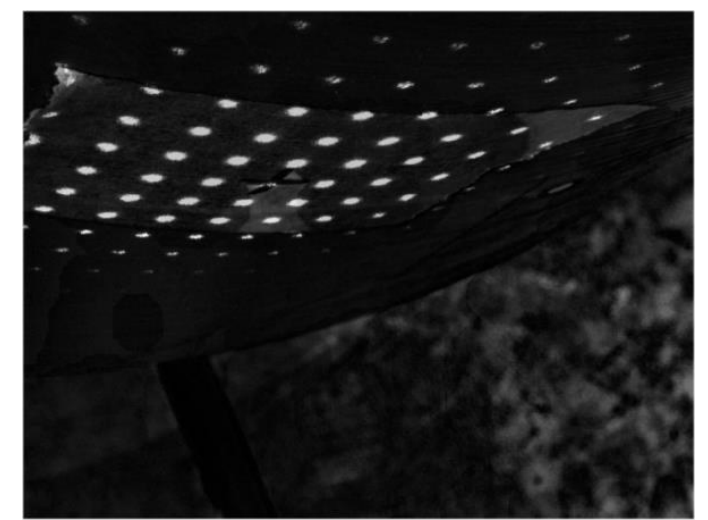

Fig. 8 The result of using Top-Hat operation 
Changing the image in shades of gray to a binary image allows image segmentation and extraction of objects (markers) from the background (Fig. 9) (Roy et al., 2014).

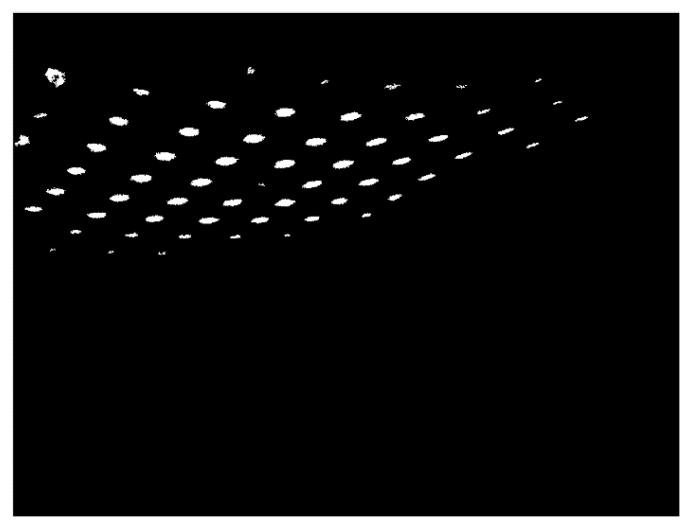

Fig. 9 An image of a binary marker grid

Depending on the value of the binarization threshold, the object will be separated from the scene with different accuracy. With even exposure of photos, it is possible to choose such a binarization threshold value that will ensure high efficiency of separating objects from the background. There is also a method that allows adaptive image thresholding (Bradley \& Roth, 2007). The above-mentioned operations on measuring photos describe the following equations (Malina \& Smiatacz, 2005):

- $\quad$ erosion of set $A$ by $B$ where $B$ is a structuring element:

$$
A \ominus B=\bigcap_{b \in B}(A+b)=\left\{x, y: B_{x y} \subseteq A\right\}
$$

where:

$A$ - image,

$B$ - structuring element,

$b$ - a common part of the sets,

$x, y$-image coordinates,

- dilatation of set $A$ through $B$ where $B$ is a structuring element:

$$
A \oplus B=\cup_{b \in B}(A+b) \quad=\left\{x, y: B_{x y} \cap A \neq \emptyset\right\}
$$

- opening of object $\mathrm{A}$ with element $\mathrm{B}$ :

$$
A \circ B=(A \ominus B) \oplus B
$$

- Top-Hat operation:

$$
T H=A-(A \circ B)
$$

where:

TH- output image,

- binarization:

$$
\begin{array}{r}
0 \operatorname{dlaT} T(x, y)<T \\
1 x, y)=\sum_{x, y} 1 \operatorname{dlaTH}(x, y) \geq T
\end{array}
$$

where:

$h$ - output image,

$\mathrm{TH}$ - input image,

$T$ - threshold. 
The resulting pair of binary images show markers with numerous interferences (Dudzik \& Sochacka, 2018). To get rid of them and extract sought after features of the object, the bwareaopen function is used, which removes small objects from the binary image (Fig. 10).

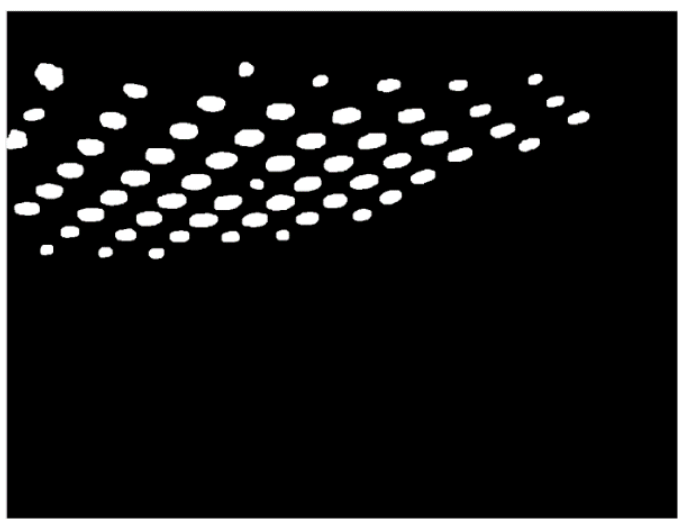

Fig. 10 Binary image after removing impurities

The argument of this function is the maximum size of the object to remove. This function works on planting the opening of an object with a small kernel. We can also use the imclearborder function, which removes objects connected to the image border. Because as a result of binarization, the contour of markers is usually jagged, to make it smooth again, expansion joints are used, followed by erosion operations (closing) - Fig. 11:

$$
A \cdot B=(A \oplus B) \ominus B
$$

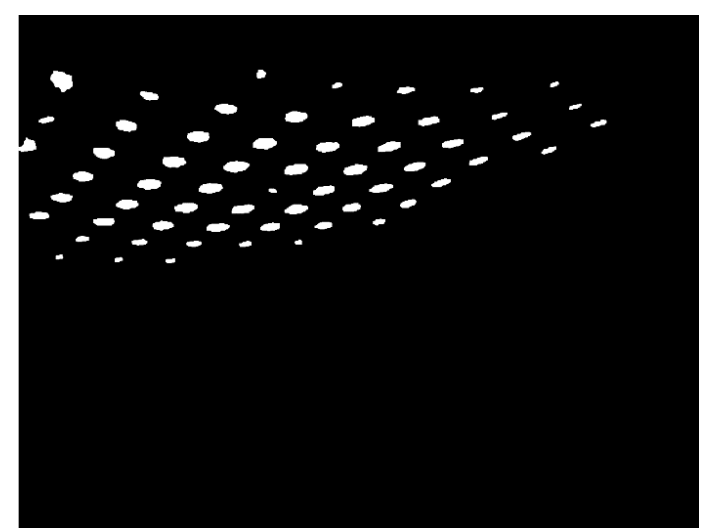

Fig. 11 The result of using morphological operations on a binary image with a separate marker grid

In this way, markers in the form of regions with a value of 1 on the binary image were separated from the pair of measurement photos. The number of regions does not have to be the same for both pictures. It is possible that one of the markers was registered by only one camera or as a result of segmentation of measurement photos, the image of some marker was not extracted from the photo. The position of the regions in the photo is described by the coordinates $(x, y)$ expressed in pixels. These coordinates describe the location of the geometric centre of the region. Automatic indexation numbers individual regions according to the increasing value of the $x$ coordinate (Fig. 12). 

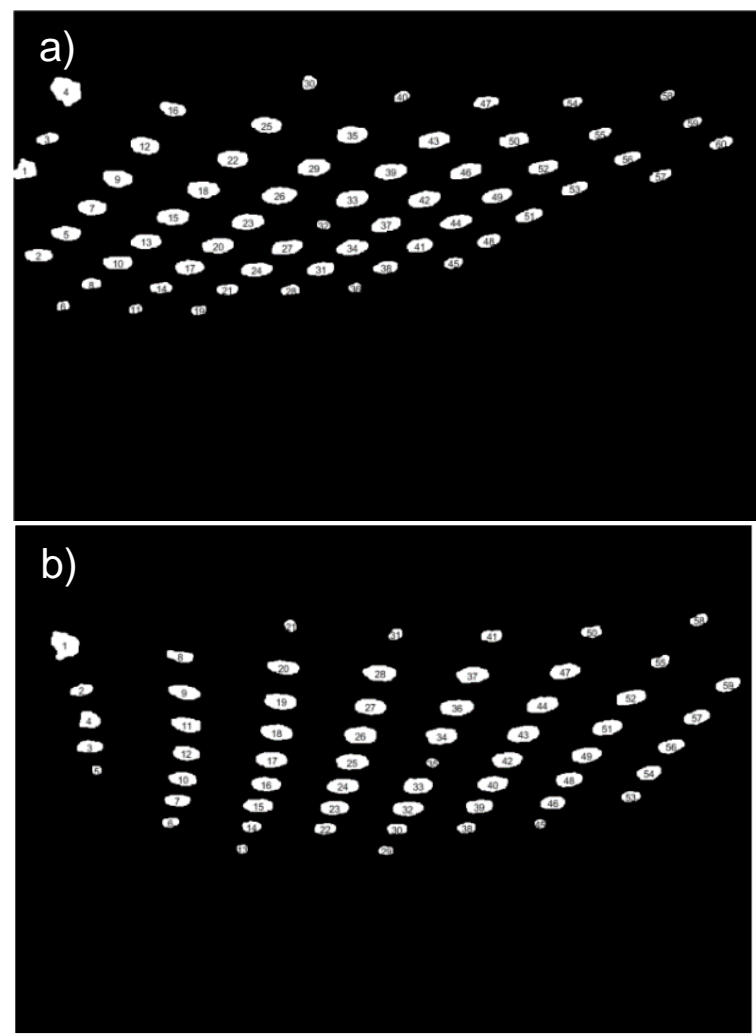

Fig. 12 The default way to index objects representing markers: a) image from the left camera, b) image from the right camera

Because the grid of markers in the scene of the analyzed images is characterized by different spatial orientation, automatic indexation does not assign the corresponding marker images to a pair of measuring images. It was, therefore, necessary to develop an algorithm for finding stereo correspondence between the markers extracted in the pictures.

\section{MIDDLE MARK DETECTION}

The developed marker indexation method is based on the search on both measurement photos of their images in the environment of the previously identified marker on the measurement photos. The reference point is the middle mark in the form of a cross. From him begins the process of identifying the markers in the measurement photos.

The original image shows a middle mark (in the form of a cross) that marks the midpoint of the marker grid pattern. The centre point has just been chosen as the reference point because the marker images in the images near the centre of the marker grid pattern are the most concentrated. As we move away from this centre, the density of these images decreases. It is also most likely that these markers in this area were recorded on both measurement images.

The middle mark is detected on the recorded measurement images during image segmentation (Chen, Artieres \& Denoyer, 2019). Preliminary processing of measurement data begins, as for other markers, from the conversion of photos into eight-bit images in shades of gray. Then the contrast of the images is changed by the histeq function which changes the histograms of the images in such a way that they are maximally flat (histogram equalization) to emphasize 
the marker under consideration (Fig. 13). This operation is described by the equation (Tadeusiewicz \& Korohoda, 1997):

where:

$$
\begin{aligned}
& H i S_{s k}=\frac{1}{M N} \sum_{k=0}^{g} H i s(k) \\
& m(g)=H i s_{s k}(g)(G-1)
\end{aligned}
$$

$G$ - number of brightness levels,

$M N$ - source image sizes,

His - digital image histogram,

Hissk-cumulative histogram.

Initial processing ends with the removal of distortion caused by optical system defects.
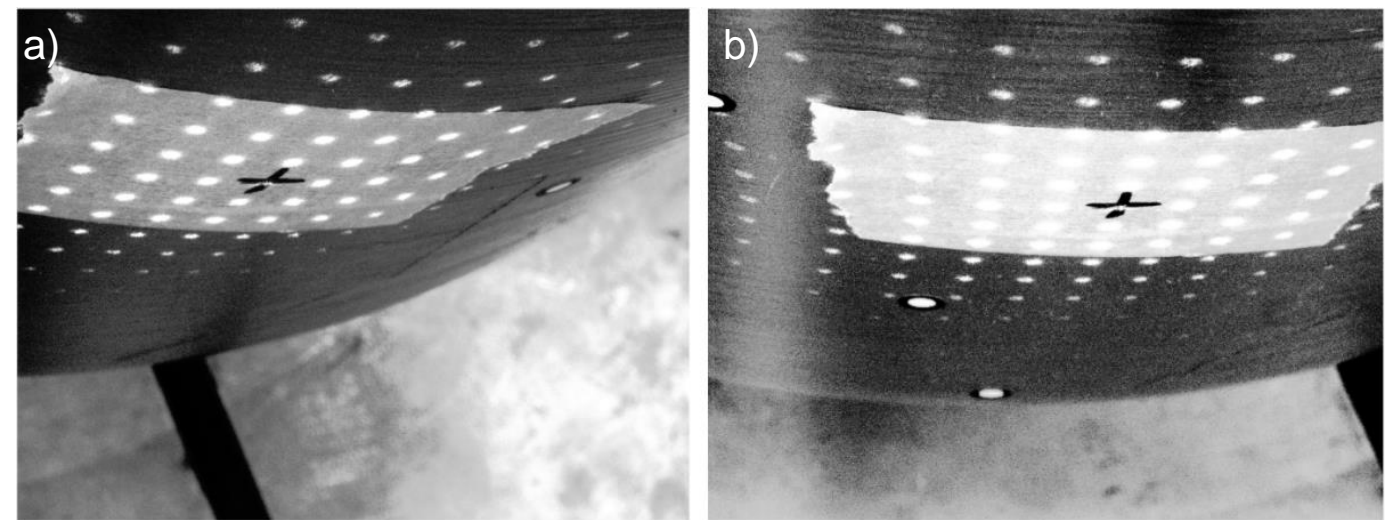

Fig. 13 Application of the histeq function on photos:

a) from the left camera, b) from the right camera

This time, morphological operations are used in reverse order to extract the middle mark, which is one of the darkest regions in the pre-processed images. The processed image is subtracted from the separated background by extracting the middle mark (Fig. 14), (Fig. 15) \& (Fig. 16).

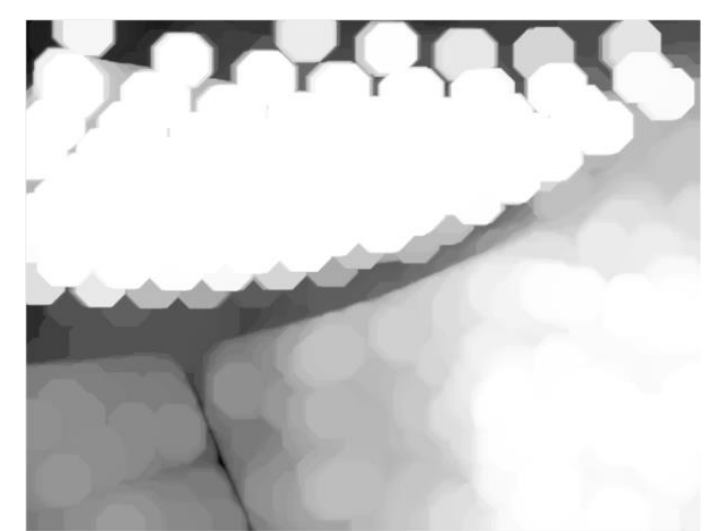

Fig. 14 The result of applying dilation operations $n$ monochrome images during middle mark detection

A Bottom-Hat transformation is performed, which is described by the equation:

$$
B H=(A \bullet B)-A
$$

where:

$A$ - input image, 
$B$ - structuring element, $\mathrm{BH}$ - output image.

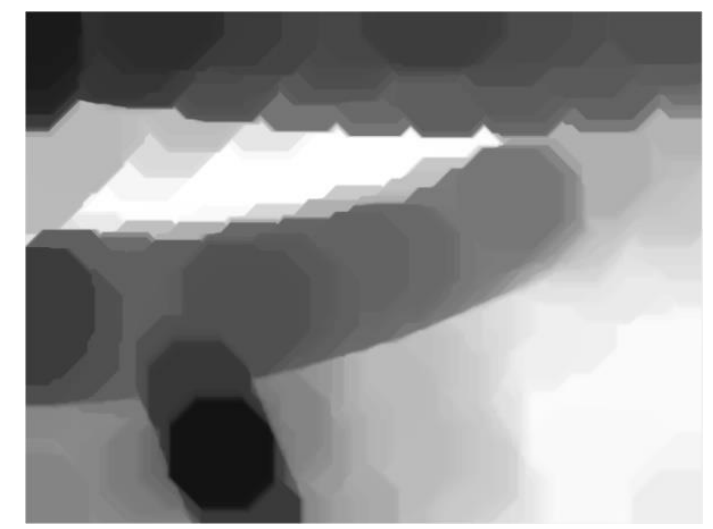

Fig. 15 The result of applying erosion on monochrome images during middle mark detection

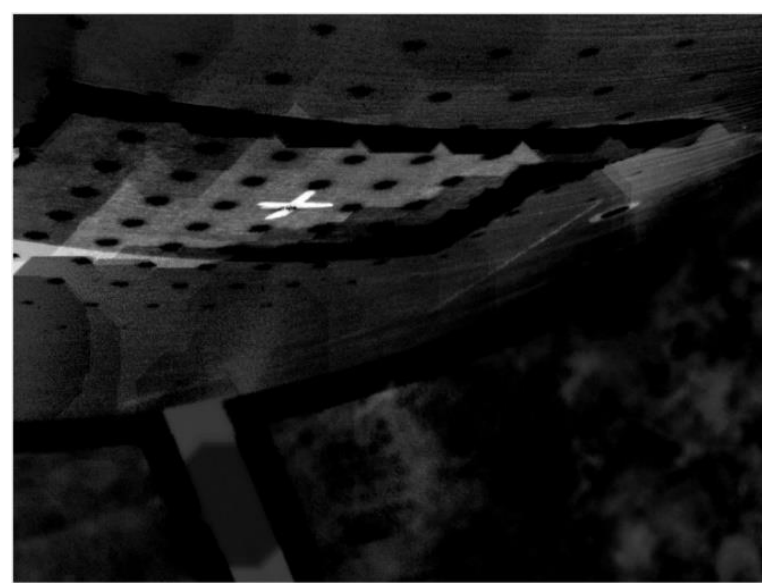

Fig. 16 The result of applying the Bottom-Hat operation during middle mark detection

Then the resulting images are changed to binary images. Unfortunately, for each photo, the binary threshold enabling the desired segmentation of the tag is different. Adaptive thresholding can help with finding the correct threshold. Numerous distortions characterize the separated object due to interference caused by image processing (Fig. 17).

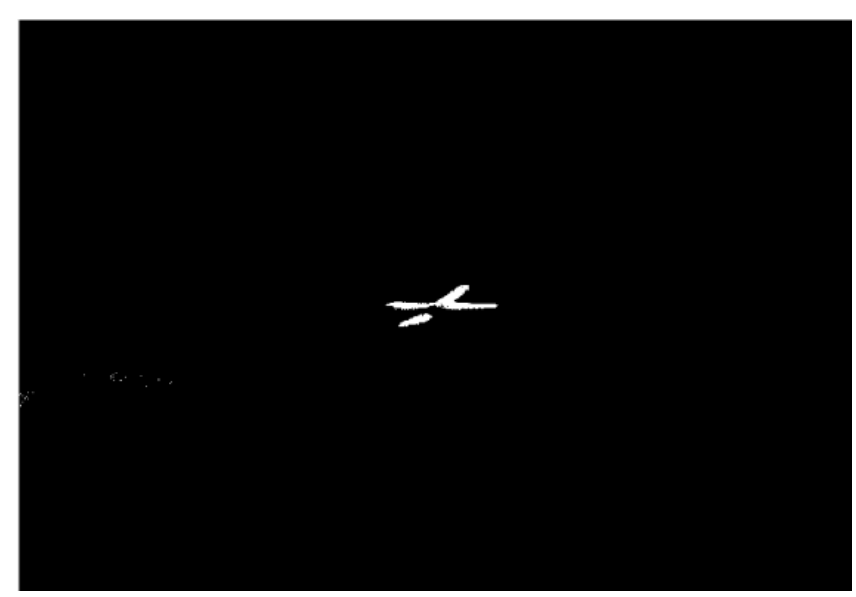

Fig. 17 Binary image with the separated middle mark 
In order to emphasize the features of the object, similar procedures are used for the extraction of the marker grid (Fig. 18) \& (Fig. 19). The position of the separated region representing the marker is described by the $x, y$ coordinates (in pixels) that determine the geometric centre of this region.

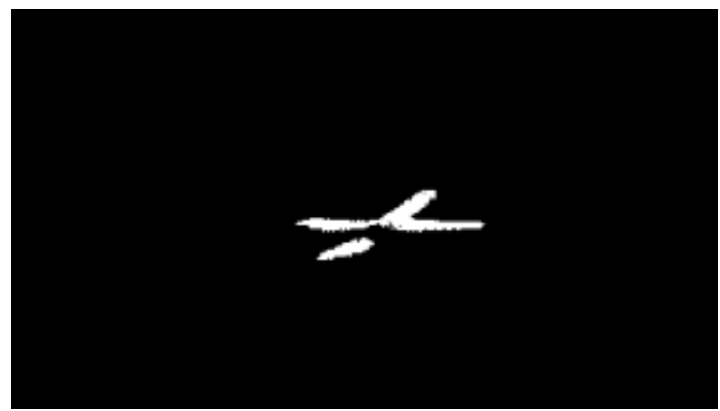

Fig. 18 Binary image of the middle mark after removal of impurities

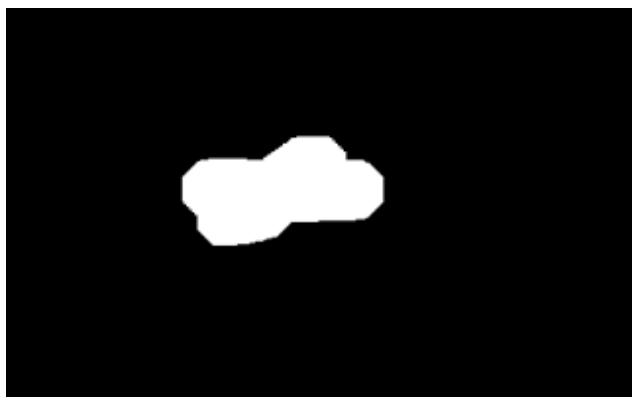

Fig. 19 The result of using morphological operations on a binary image with a separate middle mark

Markers on pre-processed images can also be identified using an object detector (Szymczyk, 2008). In Matlab, by setting parameters such as the number of iterations or the type of object features, it is possible to train such a detector using the trainCascadeObjectDetector function. For this purpose, using the Matlaba Image Labeler application, indicate on the sample of photos the object of interest (middle mark) in various positions and prepare a catalogue with photos on which the object is not located. A properly trained detector detects the object on given images with high efficiency and gives the coordinate values of the centre of the detected object.

Knowing the coordinates of all visible markers and the middle mark, we can proceed to indexation of markers registered on both measuring images. Index 1 is assigned to the marker that overlaps with the middle mark.

\section{DETERMINING THE PATTERN DISTORTION AND DETERMINING THE DIRECTION OF THE MARKER SEARCH}

The marker grid applied to the side of the cutting head, depending on the location and orientation of the cameras relative to it during the acquisition of measuring photos, has a different shape. In order to be able to find the stereocorrespondency of individual markers on a pair of measuring photos, it is necessary to determine how the pattern in a given photo is distorted (Fig. 20). How markers decompose can be determined by middle mark distortion 
(Ashburner \& Friston, 1997). Depending on whether the middle mark is rotated, skewed or rotated and skewed, the marker grid decomposes in a certain way.

a)

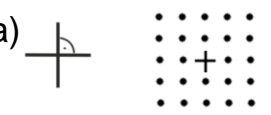

b)

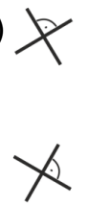

c)

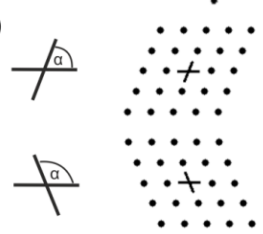

Fig. 20 Distortion of the marker grid and the middle mark: a) image without distortion, b) rotated image, c) tilted image

The developed method determines the rotation of both straight sections that make up the middle mark cross in the coordinate system of the measuring images. In the binary image with the separated marker, the pixels with the value 1 with the smallest $x$ and $y$ coordinate value and, with the largest $x$ and $y$ value (Fig. 21) that are looking for the extreme pixel arms of the middle mark cross are searched in order.

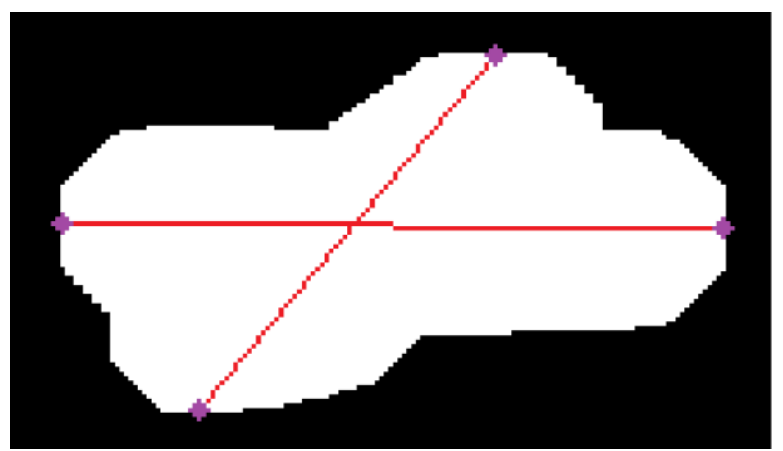

Fig. 21 Method of determining the distortion of the middle mark in the form of a cross based on the position of its arms

Having averaged values of the $x$ and $y$ coordinate for each group of pixels, the lengths of individual segments making up the marker are calculated, and their rotation on the image (Fig. 22). The angle of inclination of the horizontal arm $\alpha_{P 1}$ is determined from the length of the projection of this section on the $\mathrm{X}$-axis and the length of its projection on the $\mathrm{Y}$-axis. The inclination angle of the horizontal section of the $\alpha_{P 2}$ arm is calculated based on the length of the projection of this section on the $\mathrm{X}$-axis and the length of its projection on the $\mathrm{Y}$-axis:

$$
\begin{aligned}
& \alpha_{P 1}=\operatorname{arctg}\left(\frac{y_{12}-y_{11}}{x_{12}-x_{11}}\right) \\
& \alpha_{P 2}=\operatorname{arctg}\left(\frac{y_{22}-y_{21}}{x_{22}-x_{21}}\right)
\end{aligned}
$$




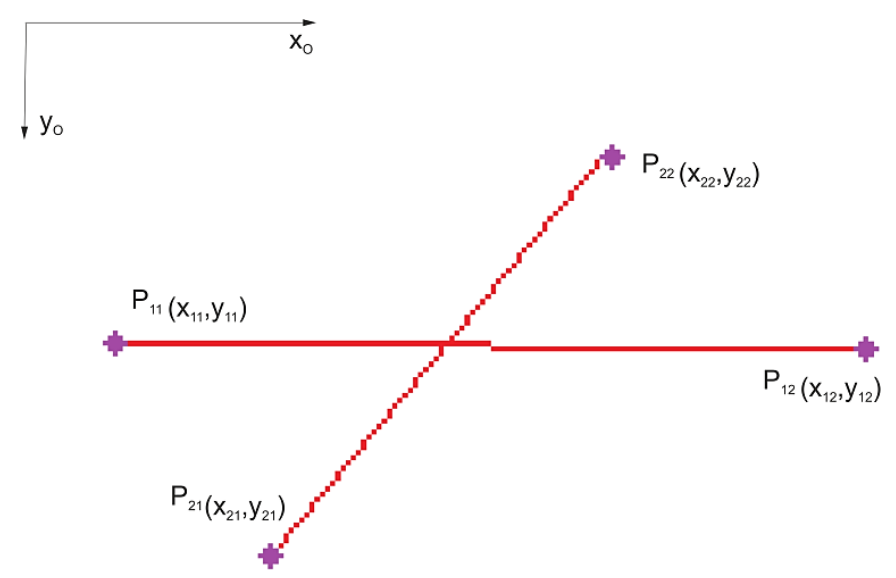

Fig. 22 Coordinates of the average values of extreme pixels of the middle mark cross arms

If the angle between the sections is approximately a right angle, the marker grid is not skewed. Then, if the angle of the remaining segments is approximately 0 and $90^{\circ}$, the marker grid is also not rotated. Based on this, four search directions are determined along which images of subsequent markers will be searched for in certain areas (Fig. 23):

$$
\begin{aligned}
& \beta_{h 1}=\alpha_{P 1} \\
& \beta_{w 1}=\alpha_{P 2} \\
& \beta_{h 2}=\alpha_{P 1}+180^{\circ} \\
& \beta_{w 2}=\alpha_{P 2}+180^{\circ}
\end{aligned}
$$

where:

$\beta_{h 1}-$ Search direction along the horizontal segment with the turn directed along the $\mathrm{X}$-axis of the image coordinate system, $\beta_{w 1}-$ Search direction along the vertical section with the turn directed opposite to the $\mathrm{Y}$-axis of the image coordinate system,

$\beta_{h 2}$ - Search direction along the horizontal segment with the turn directed opposite to the X-axis of the image coordinate system,

$\beta_{w 2}-$ Search direction along the vertical section with the turn directed along to the $\mathrm{Y}$-axis of the image coordinate system.

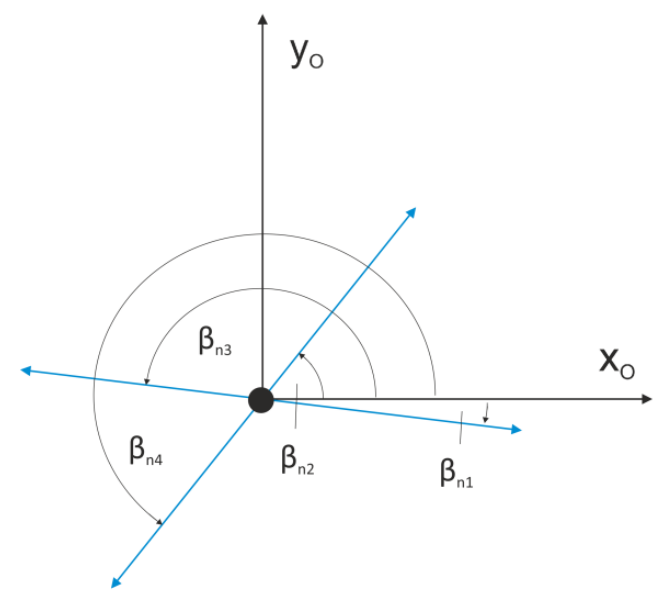

Fig. 23 Designated search directions (blue lines) 
The size and location of a given area are closely related to the given search direction, and the density of the marker grid displayed on the side surface of the cutting head. They are determined based on the length of the arms of the central marker cross-registered in the pictures, assuming that their length corresponds to a certain extent to the distance between the markers along the given direction. If we use an object detector to detect a middle mark, we can use the estimateGeometricTransform function to estimate the geometric transformation of the detected object and thus determine the direction of the marker search.

\section{INDEXING OF CORRESPONDING MARKER IMAGES ON MEASUREMENT PHOTOS}

Starting from the reference point (middle mark in the form of a cross) on measuring photos, the procedure for indexing following pairs of marker images looks for subsequent markers along one of the directions determined based on the distortion of the middle mark (Fig. 24).

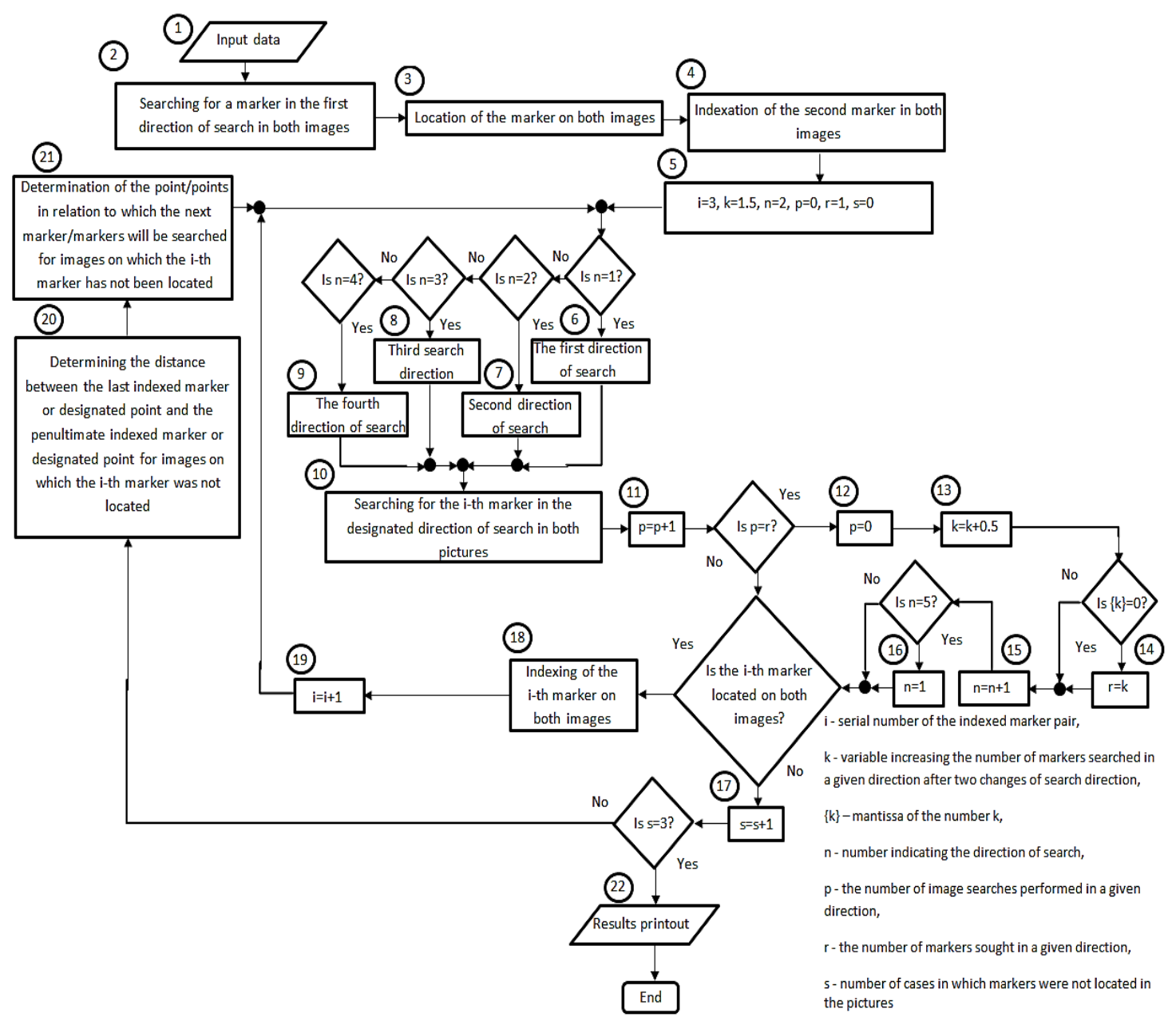

Fig. 24 The algorithm of the procedure for automatic detection and indexing of subsequent markers

Another marker is sought along the horizontal segment with a phrase consistent with the return of the $\mathrm{X}$-axis of the photo coordinate system, such that the value of the $x$ coordinate is higher than the $x_{1}$ coordinate of the reference point and also the smallest: 


$$
\begin{aligned}
& \left\{x_{j} \in X: x_{j}>x_{1}\right\} \\
& \forall x_{j} \in X: x_{2} \leq x_{j}
\end{aligned}
$$

where:

$x_{j}-\quad$ the $x$ coordinate of the marker found higher than $x_{1}$ along the first of the search directions,

$X$ - set of coordinates $x_{j}$,

$x_{1}$ - the $x$ coordinate of the first indexed marker,

$x_{2}$ - the $x$ coordinate of the second indexed marker.

The process is implemented programmatically checking the location of each of the markers in addition to those previously indexed. If a marker is found in both pictures, it is marked with the next number (Fig. 25).

a)

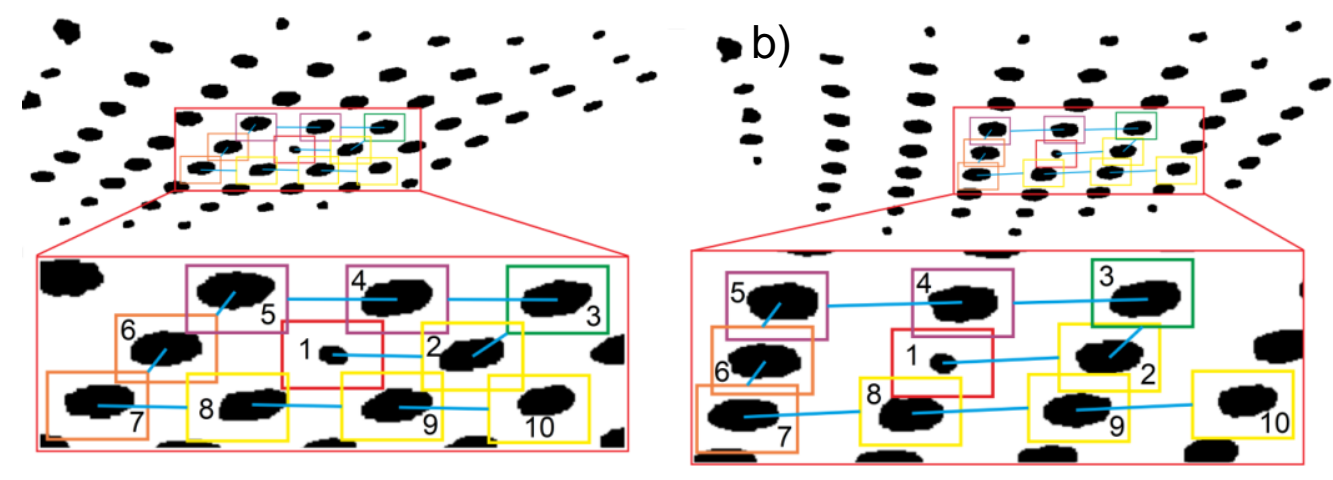

C)

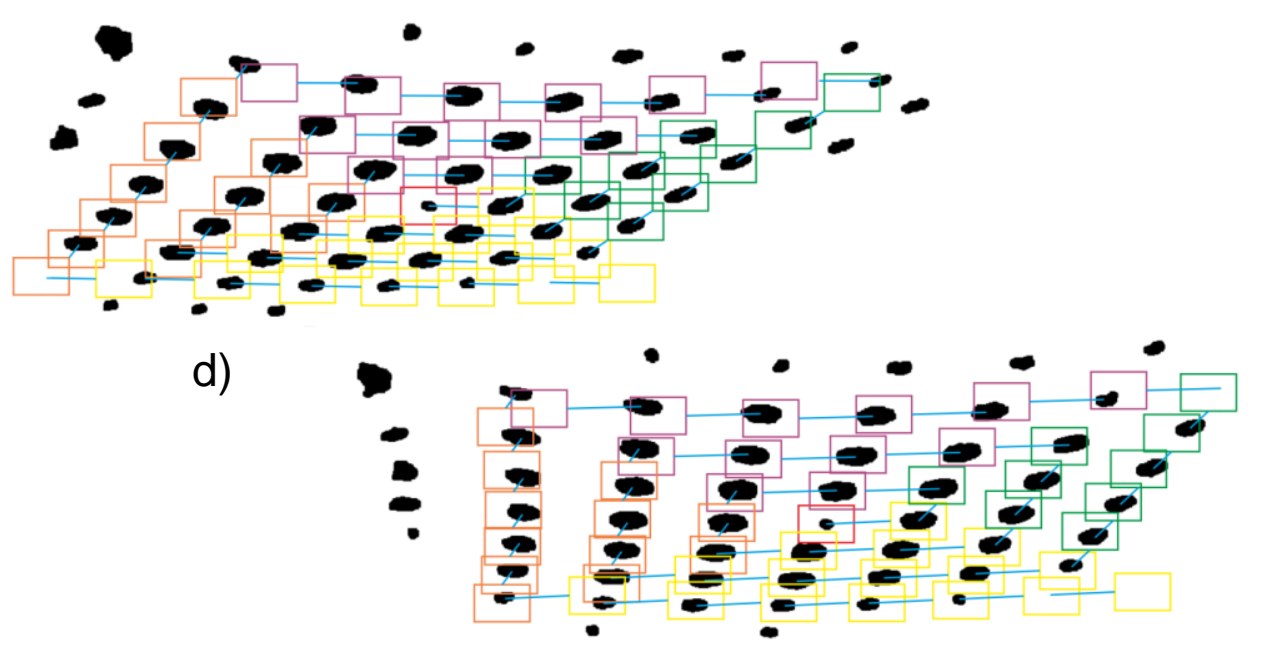

Fig. 25 Steps of detecting and indexing markers in the measurement photo:

a) the beginning of the procedure for the photo from the left camera,

b) the beginning of the procedure for the photo from the right camera,

c) the end of the procedure for the photo from the left camera,

d) the end of the procedure for the photo from the right camera

Then, concerning the previously classified pair of marker images, the next pair of markers is searched for in the analyzed images. This time next marker images in the photos will be searched along the vertical segment with the opposite turn to the $\mathrm{Y}$-axis return of the photo coordinate system, such that they coordinate value is smaller than the $y$ value of the last indexed marker and also the largest:

$$
\begin{aligned}
& \left\{y_{j} \in Y: y_{j}<y_{2}\right\} \\
& \forall y_{j} \in Y: y_{i+1} \geq y_{j}
\end{aligned}
$$


where:

$y_{j}-\quad$ the $y$ coordinate of the marker found higher than $y_{1}$ along the second of the search directions,

$Y$ - set of coordinates $y$,

$y_{2}$ - the $y$ coordinate of the second indexed marker,

$y_{i+1}$ - the $y$ coordinate of the next indexed marker.

When we find a pair of marker images, they are marked with a sequential number. After each change of the search direction twice, the number of searched objects in a given direction increases by 1 . The next pair of marker images is searched for along the horizontal segment with the opposite turn to the $\mathrm{X}$-axis return of the image coordinate system so that the $x$ coordinate is smaller than the $x$ coordinate of the previous indexed pair of markers and also the largest:

where:

$$
\begin{aligned}
& \left\{x_{j} \in X: x_{j}<x_{i}\right\} \\
& \forall x_{j} \in X: x_{i+1} \geq x_{j}
\end{aligned}
$$

$x_{j}-\quad$ the $x$ coordinate of the marker found smaller than $x_{i}$ along a third of the search directions,

$X$ - set of coordinates $x_{j}$,

$x_{i}$ - the $x$ coordinate of the i-th indexed marker,

$x_{i+1}$ - the $x$ coordinate of the next indexed marker.

The pair of marker images found receives the next number. First, markers are sought near the centre of the grid, because these markers are in the place where the given pick holder is to be mounted. The markers are indexed in an ordered way around the middle mark. Along the vertical segment with a turn corresponding to the return of the $\mathrm{Y}$-axis of the photo coordinate system, a marker is sought such that the y coordinate value is higher than the $y$ coordinate value of the last indexed marker and at the same time the smallest. Subsequently found pairs receive consecutive numbers.

$$
\begin{aligned}
& \left\{y_{j} \in Y: y_{j}>y_{i}\right\} \\
& \forall y_{j} \in Y: y_{i+1} \leq y_{j}
\end{aligned}
$$

where:

$y_{j}-\quad$ the $y$ coordinate of the marker found smaller than $y_{i}$ along a fourth of the search directions,

$Y$ - set of coordinates $y_{j}$,

$y_{i}$ - the $y$ coordinate of the i-th indexed marker,

$y_{i+1}$ - the $y$ coordinate of the next indexed marker.

Subsequent pairs of marker images will be searched again in the first designated search direction until the next three markers are classified, and the search direction and rules are not changed. The process continues to index all objects. To prevent indexing of objects that do not form stereo pairs, we are only looking for more objects in a specific environment from the previously indexed marker. Due to the round shape of the side surface of the cutting head and the laser distortion of the projection device, as the distance from the centre of the grid, 
the distance between markers increases. As we move away from the centre of the marker grid during indexing, the distance at which we expect to locate the next marker increases.

It happens that some marker images are deleted as a result of processing measuring images. This applies especially to markers distant from the centre point. If during the search for the next pair of marker images, it is located on only one photo, the coordinates of the found marker image serve as a reference point in the search for the next marker image in this photo. For the photo for which the marker image has not been located to determine the reference point, the difference between the coordinates of the last and the penultimate object among the located objects in the given photo is added to the coordinates of the last located marker.

If, during indexing of markers, there is a situation three times where the marker is not located, but on one photo from the pair, then the algorithm for indexing the pair of markers ends its operation.

During the acquisition, the middle mark should be located on the measurement photos as close as possible to the centre of the marker grid. Circles around the marker turn around as we look for more markers. This location of the marker relative to the rest of the markers provides the ability to identify more markers.

\section{SAMPLE RESULTS OF THE APPLICATION OF THE AUTOMATIC MARKER IDENTIFICATION METHOD}

The developed method has been experimentally tested. Measurement photos were taken from the KUKA KR16-2 robot control system. For this purpose, a stereovision head equipped with two KUKA MXG20 cameras with a convergent orientation was used. Markers on the side surface of the cutting head are applied using a laser projection device placed on a tripod. The downloaded images were further processed and analyzed on a computer in the Matlab environment (using the libraries of the Image Processing Toolbox package) by the discussed procedure.

By segmenting pre-processed images for extraction of a marker and marker grid, and using the developed method of indexing corresponding objects on pairs of measurement images, the indexation of markers was made for three sample pairs of measurement images (Fig. 26). The recorded images show the side areas of the cutter head with different geometries. 


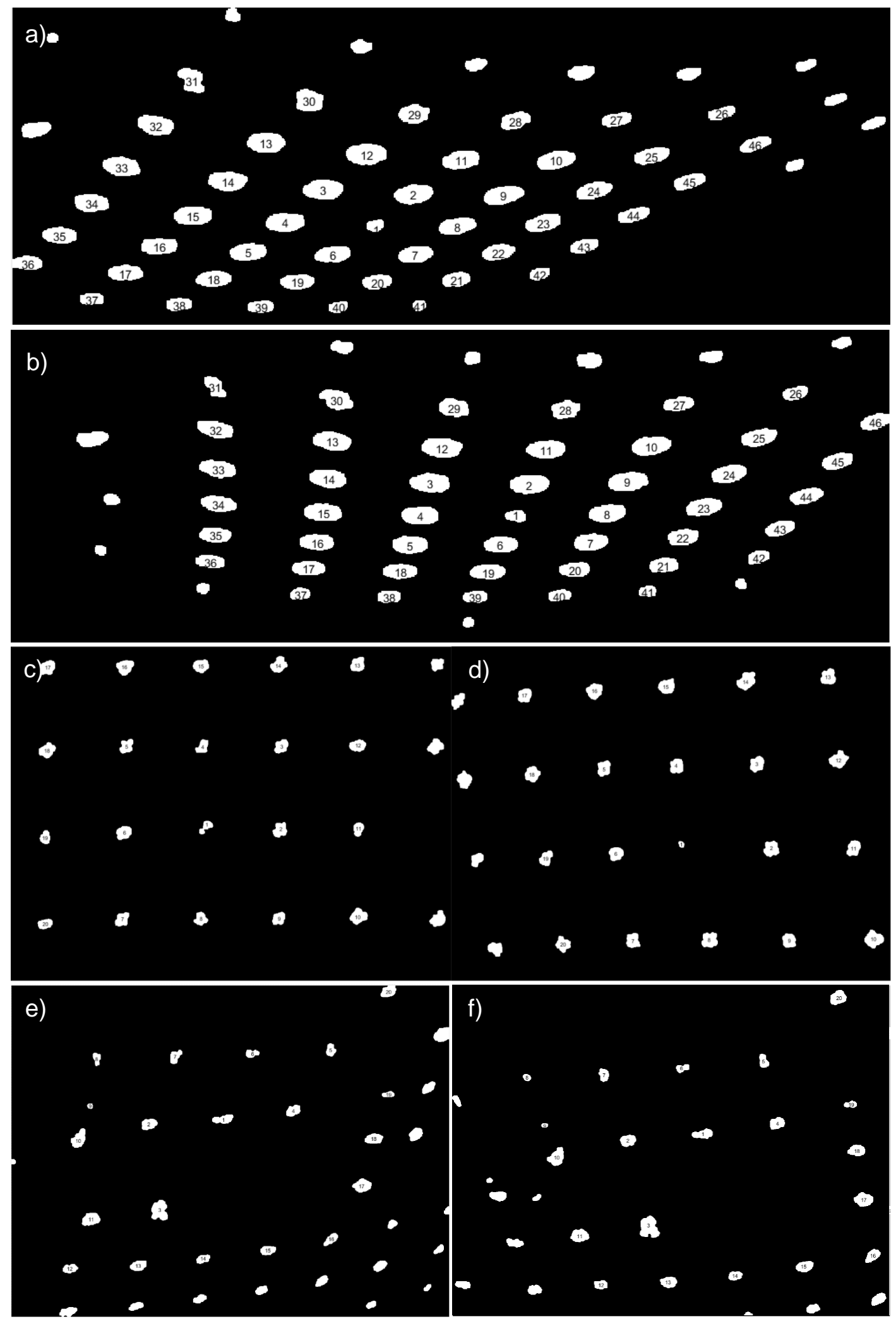

Fig. 26 Sample results of the application of the automatic marker identification method on measuring photos: a) left picture of the first pair, b) right picture of the first pair, c) left picture of second pair, d) right picture of second pair, e) left picture of the third pair, f) right picture of the third pair

\section{CONCLUSIONS}

The developed method of indexing marker images on pairs of measuring images enables the identification of corresponding pairs of marker images on images 
forming a stereo pair. This method is useful when creating three-dimensional models based on photos taken with cameras in a convergent setting. The use of triangulation requires the indication of corresponding points on a pair of photos. The recorded images show the side surface areas of the cutting head with different geometries.

Markers based on the centre coordinates can be automatically searched for and counted on measurement photos in different directions, and in order depending on how the marker grid is arranged, the type of markers and marker. The algorithm will perform its task even if during the processing of a pair of measuring photos a different number of marker images were separated or there are slight deformations on the photographed side surface of the working unit of the mining machine.

Thanks to three-dimensional reconstruction of markers separated only from photos, a spatial model of only interesting objects is obtained, for further processing of the acquired data. Further work will be conducted to provide the developed algorithm with the ability to find stereo-correspondence between the images of markers if:

- the photographed surface of the side surface of the working unit of the mining machine is heavily deformed,

- the number of separated markers as a result of image processing is not the same for both measurement images.

\section{REFERENCES}

Ashburner, J. and Friston, K.J. (1997) Spatial Transformation of Images. London.

Bräuer-Burchardt C. Voss K. and Schiller F. (2000) 'Automatic Lens Distortion Calibration Using Single Views'. in Sommer, G. Krüger, N. Perwass, C. (ed) Mustererkennung 2000. Informatik aktuell. Berlin, Heidelberg: Springer, pp. 187194.

Bradley, D. Roth, G. (2007) 'Adaptive Thresholding Using the Integral Image', Graphics Tools, 12(2), pp. 13-21.

Cheluszka, P. Nocoń, M. (2015) 'Robotised digitalisation technology of roadheader working units for the purpose of manufacturing quality control', Surface Mining, 56(6), pp. 11-23. (in Polish)

Chen, M. Artieres, T. Denoyer, L. (2019) 'Unsupervised Object Segmentation by Redrawing', Conference on Neural Information Processing Systems, NeurlPS.

Corke, P.I. (1996) Visual control of robots: High-Performance Visual Servoing. Australia: CSIRO Division of Manufacturing Technology.

Dolipski M. Cheluszka P. Remiorz E. Sobota P. Osadnik J. (2010) 'Manufacturing processes of cutting machine working units using robotic technologies' in Mikołajczyk, T. (ed) CAX'2010 Group work. Bydgoszcz: Wydawnictwo Uczelniane Uniwersytetu Technologiczno-Przyrodniczego, pp. 85-90. (in Polish)

Dudzik, S. Sochacka, O. (2018) 'Application of local progression methods for detecting defects using active thermography', Intercollegiate Metrologists Conference Zeszyty Naukowe Wydziału Elektrotechniki i Automatyki Politechniki Gdańskiej, 59(8), pp. 43-46. (in Polish)

Ewald, M. (2009) 'Content-Based Image Indexing and Retrieval in an Image Database for Technical Domains', Transactions on Machine Learning and Data Mining, 2(1), pp. 3-22. 
Fisker, R. Poulsen, H.F. Schou, J. Carstensen, J.M. Garbe, S. (1998) 'Use of ImageProcessing Tools for Texture Analysis of High-Energy X-ray Synchrotron Data', Journal of Applied Crystallography, 31, pp. 647-653.

Goral, A. (2014) 'Comparison of selected marker tracking methods for video locators', Przegląd elektrotechniczny, 90(5), p97-101. (in Polish)

Jagieła-Zając, A. Cheluszka, P. (2019) 'Measurement of the pick holders position on the side surface of the cutting head of a mining machine with the use of stereoscopic vision', IOP Conference Series: Materials Science and Engineering, 679(1) [online]. Available at: https://iopscience.iop.org/article/10.1088/1757899X/679/1/012005/pdf (Accessed: 28 February 2020).

Kubiak, I. (2014) 'Algorithm for equalizing the histogram of image pixel amplitude values in the image processing process obtained from electromagnetic emission signals correlated with video signals', Przegląd Telekomunikacyjny, 7, pp. 682-686. (in Polish)

Kryjak, T. Komorkiewicz, M. (2013) 'Real-time FPGA implementation of disparity map calculation for a 3D video stream', PAK, 59(8), pp. 748-750. (in Polish)

Malina, W. Smiatacz, M. 2005 Digital image processing methods. Warszawa: Akademicka oficyna wydawnicza EXIT. (in Polish)

Raviya, K.S. Kothari, A.M. Vyas, D.V. (2014) 'Depth and Disparity Extraction Structure for Multi View Images-Video Frame- A Review', European Journal of Academic Essays, 1(10), pp. 29-35.

Roy, P. Dey, N. Dey, G. Dutta, S. Chakraborty, S. Rey, R. (2014) 'Adaptive thresholding: A comparative study', International Conference on Control, Instrumentation, Communication and Computational Technologies, p1182-1186.

Sricha, R. Khan, A. (2013) 'Morphological Operations for Image Processing: Understanding and its Applications', NCVSComs conference proceedings, 13, pp. 17-19.

Szymczyk, T. (2008) 'Pattern matching method in image recognition - limitations, problems and modifications of the method', Automatyka, 12(2), pp. 449-462. (in Polish)

Tadeusiewicz, R. Korohoda, P. (1997) Computer analysis and image processing. Kraków: Wydawnictwo Fundacji Postępu Telekomunikacji. (in Polish)

Wojciechwski, P. Bal, A. (2012) 'Method of finding cells and counting foci of histone $\mathrm{Y}^{-}$ H2AX in images for the purpose of double-stranded DNA crack detection', PAK, 58(4), pp. 387-390. (in Polish)

Wrobel, Z. Koprowski, R. (2004) Image processing practice with tasks in the program Matlab. EXIT. (in Polish)

Yang, W. Zhang, G. Zhang, X. (2019) 'Infrared LEDs-based pose estimation with underground camera model for Boom-type roadheader in coal mining', IEEE, 7, pp. 33698-33712.

Zhang, Y. (2009) 'Image processing using spatial transform', International Conference on Image Analysis and Signal Processing, pp. 282-285.

Żorski, W. Samsel, P. 2009 'Segmentation of color images with irregular patterns' Biuletyn instytutu automatyki i robotyki, 26, pp. 45-65. (in Polish) 


\begin{abstract}
.
Ensuring the compliance of the finished product with the project during the manufacturing of cutting heads/drums of the mining machines, largely determines the efficiency of rock mining, especially hard-to-cut rocks. The manufacturing process of these crucial elements of cutting machines is being robotized in order to ensure high accuracy and repeatability. This determines, among others the need to assess in real-time the degree of the approach of pick holders positioned by the industrial robot to the side surface of the working unit of the cutting machine in their target position. This problem is particularly important when in the manufacturing process are used the bodies of decommissioned cutting heads/drums, from which old pick holders have been removed. The shape and external dimensions of these hulls, unless they are subjected to regeneration, may differ quite significantly from the nominal ones. The publication, on the example of a road header cutting head, presents the procedure for automatically identifying and indexing markers displayed on its side surface, recorded on measuring photos by two digital cameras of a 3D vision system. Experimental research of the developed method was carried out using the KUKA VisionTech vision system installed on the test stand in the robotics laboratory of the Department of Mining Mechanization and Robotization at the Faculty of Mining, Safety Engineering and Industrial Automation of the Silesian University of Technology. Data processing was carried out in the Matlab environment using the libraries of the Image Processing Toolbox. The functions provided in this library were used in the developed algorithm, implemented in the software. This algorithm allows automatic identification of markers located in the images of the side surface of the cutting head. This is the basis for determining their location in space. The publication presents a method of segmenting images recorded by cameras into homogeneous areas. The method of separating interesting areas from the image by comparison to the pattern was presented. Also shown is the method of the automatic numbering of mutually matching pairs of markers on photos from two cameras included in the vision system depending on the spatial orientation of the marker grid in the measuring images.
\end{abstract}

Keywords: the working unit of mining machine, measurement, stereovision, image processing, robotic technologies 